\title{
Can pancreatic tissue cause haemoptysis?
}

The authors declare no financial disclosure

\begin{abstract}
A mature teratoma is a tumour of primary germ cells. It is often found in the mediastinum. The authors describe a case of a young man who demonstrated haemoptysis as the only symptom of a mediastinal tumour.

The tumour was removed operatively, sent for histopathological examination and immunohistochemistry. The removed tumour was a mature teratoma including elements of the pancreas. The authors revealed the presence of trypsin in the pancreatic acinar cells.

The proteolytic activity of the tumour was taken as the cause of haemoptysis because of enzymatic erosion of lung tissue intimately attached to the tumour.

In such cases surgical removal saves life of patients.
\end{abstract}

Key words: mediastinal teratoma, pancreatic tissue, haemoptysis, proteolysis

Pneumonol Alergol Pol 2016; 84: 222-224

\section{Introduction}

Primary germ cell tumours account for $10-15 \%$ of all tumours in the mediastinum. They are usually observed during the third or fourth life decade. They are most often found in the anterior mediastinum, where primordial germ cells strayed during embryonic development [1]. The most common are mature teratomas. The malignant transformation occurs here in less than $1 \%$ of cases and it is on a par with permanent growth of the tumour. The possibility of rupture is the reason for surgical intervention. Sometimes during the operation tumours are strictly adhering to surrounding organs and exhibit macroscopic invasion of adjacent structures which is explained as infection or proteolytic activity of enzymes contained in their cysts. Rupture of the tumour into surrounding structures leads to severe symptoms like purulent cough, pericardial effusion, pneumothorax, and rarely, as in our example, to haemoptysis.

\section{Case report}

A 33-year-old man was admitted urgently to our ward because of haemoptysis. He reported a similar incident six months ago. Except haemoptysis, we did not find other symptoms on physical examination. The test results were within normal limits. A chest-angio-CT scan showed irregular mass on the level of the aortic arch adhering to the mediastinum from the left. Borders were indistinct. The tumour size was 30 $\times 40 \mathrm{~mm}$ and in its centre there was a small air cavity. In the adhering pulmonary parenchyma, the radiologist described shadows called "milk glass". The air cavity in the tumour seen in CT can be interpreted as a proof of communication with the bronchial tree. The "milk-glass" shadow shows extravasations of blood into the lung [2].

The bronchoscopic examination revealed blood in the bronchus to the upper left lobe. It did not directly show a source of bleeding. 
We performed left thoracotomy. The upper lobe of the lung fused together with the mediastinum in place of the expected tumour and it demonstrated raspberry colouration here, which indicated locally extravasated blood. We cut the upper lobe with a stapler leaving its marginal piece intimately attached to the tumour. We opened the mediastinal pleura above and isolated the irregular mass of the fibrotic walls and cavity penetrating to the remaining piece of the lung. We ligated the pedicle of the tumour and removed the whole of it. The patient made an uneventful recovery from the operation and is well for more than two years now.

\section{Results}

The examination of the operation specimen showed as follows: pulmonary parenchyma with extravasations and a small nodule $(1.5 \mathrm{~cm}$ in diameter) showing a tumour of the teratoma morphology surrounded by the connective tissue capsule. Histologically the tumour contained the following: intestine, pancreas, dermoid cyst and the structure of the ciliary epithelium. We revealed the presence of trypsin in pancreatic-acinar cells using monoclonal antibodies against trypsin.

\section{Discussion}

During the last 20 years in our thoracic surgery department we removed 12 mature mediastinal teratomas. Tumours were isolated from the surrounding structures and did not contain glandular secretory type of tissue in 9 cases. Two tumours were densely adherent to the surrounding lung and rupture of one of them was a cause of infected pneumothorax. In these both cases we found postoperatively elements of the salivary glands (possibly enzymatically active).

Haemoptysis was observed only in the described case. It was the reason for urgent surgical intervention and it was the only symptom. We managed to find only a few such cases in the literature. Symptoms like cough, dyspnoea, pericardial effusion, arm, back and chest pain related mainly with tumour growth and mass effect, did not occur in our case due to a small diameter of the tumour. Increase of tumour pressure on the adjacent tissue occurs during the tumour growth, but acute symptoms like haemoptysis are caused by the destruction of the lung parenchyma and by communication with the bronchial tree. It appears to depend not on the size of the tumour. In this process a significant role may be played by

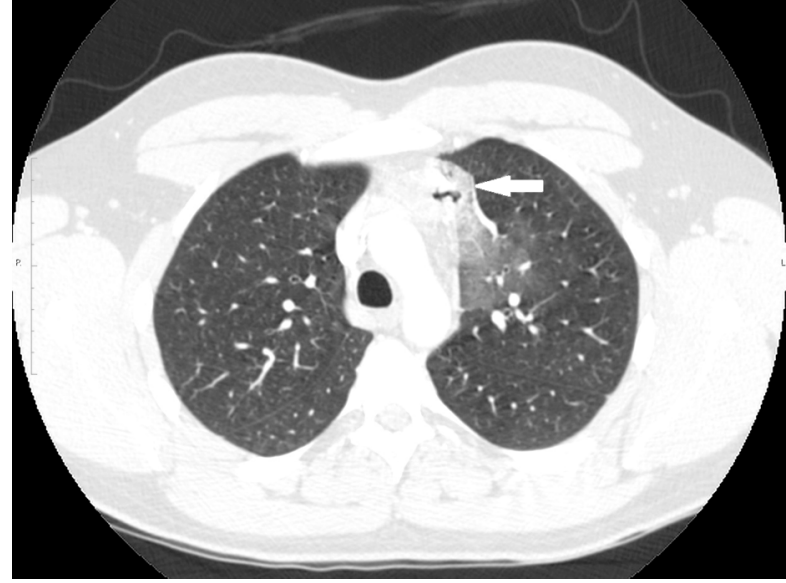

Figure 1. CT-scan: irregular mass in the mediastinum and milk-glass shadow (white arrow)

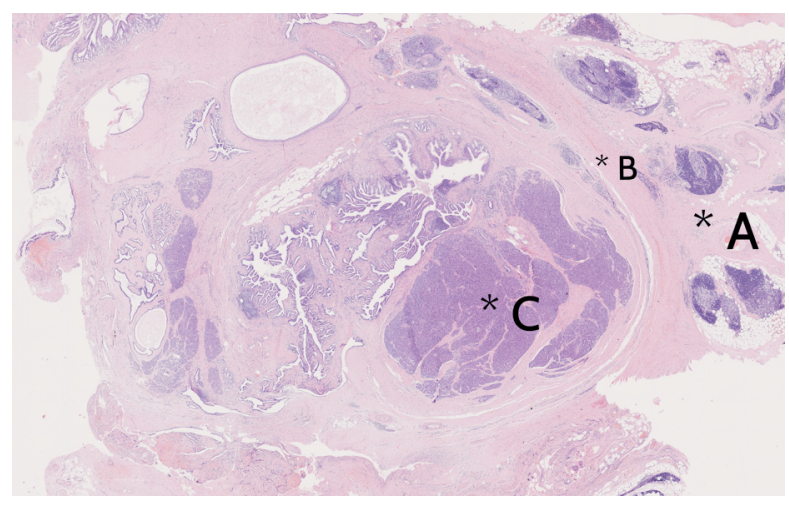

Figure 2. Section of the whole specimen: $A-$ the residual thymus; $\mathrm{B}$ - the connective tissue capsule of teratoma; $\mathrm{C}$ - the pancreas

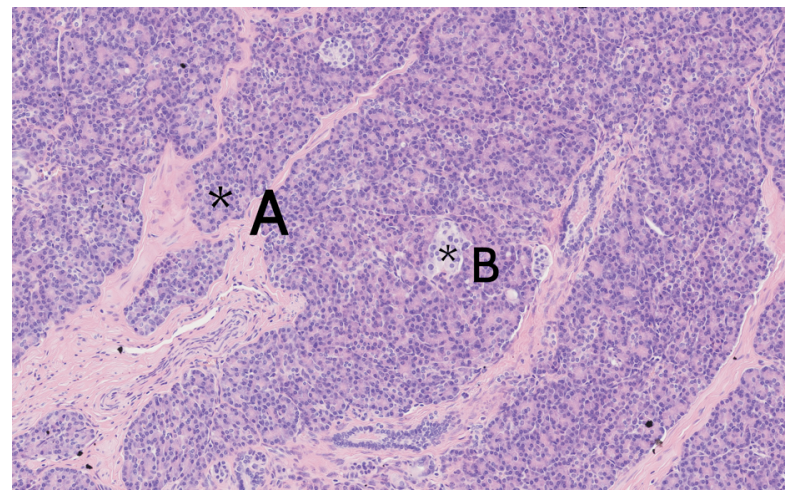

Figure 3. Zoom of the pancreas $\times 15: A-$ acinar cells; $B-$ islets

proteolysis related to the presence of a glandular secretory type of tissue, in particular pancreatic tissue with enzymatic activity [3]. We used immunohistochemistry to determine the presence of trypsin in the removed specimen and we revealed it. Presumably, enzymatic activity occurred here too. It is worth here to mentioning the authors, who have reported the possibility of the diagno- 


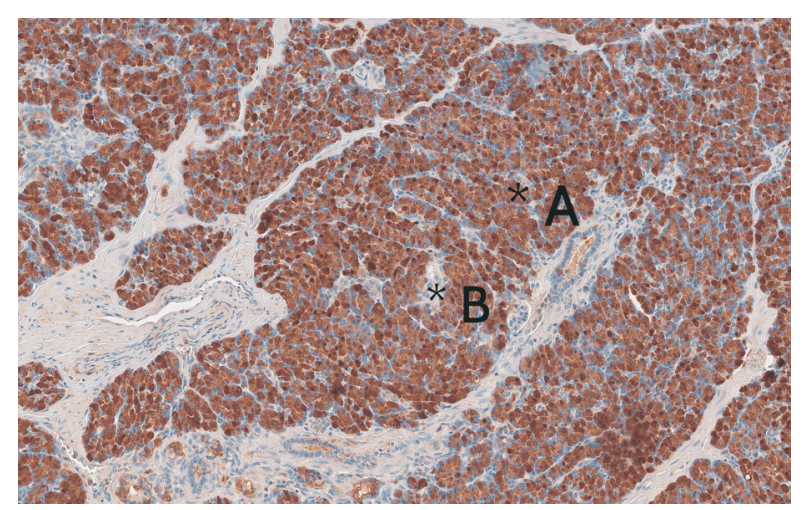

Figure 4. The pancreas labelled by immunohistochemistry: A acinar cells showing a positive reaction to the presence of trypsin (brown colour); B - islet cells showing no response to the presence of trypsin (no colour)

sis of mediastinal tumours by aspiration of the cyst contents and by demonstrating proteolytic enzymes in it [4]. We noted the analogy with necrotizing haemorrhagic pancreatitis, where damage to the elastic fibers by elastase opens the blood vessels and causes haemorrhages. Perhaps the term "erosion" as the end result of enzymatic activity would be more accurate in relation to teratoma than the frequently used term "rupture of the tumour" [2]?

Bacteriologic examination of bronchial aspirate and postoperative specimen showed no infection in our case.

Blood glucose values were in the normal range before and after the operation, but it does not mean, however, that the removed tumour was not endocrine active. In the microscopic examination of the specimen, islets could be seen. The authors showed the same precise topographic distribution of pancreatic hormones in islet cells of mediastinal teratoma that is seen in normal islets. Similarity to the physiological situation may explain the absence of hypoglycaemia in our patient [5].

\section{Conclusions}

Mediastinal teratoma can be considered as a rare cause of haemoptysis.

The risk of rupture appears to depend not only on mechanical factors like a mass or size of the tumour, but also on a type and function of tissue forming the tumour. Exocrine pancreas elements presumably played a leading role in the reported case.

Total surgical removal of mature teratoma is the treatment of choice, it stopped the bleeding and saved life of our patient, allowing hope for full recovery.

\section{Conflict of interest}

The authors declare no conflict of interest.

\section{References:}

1. Karas SM, Parissis JT, Antoniades C, Loulias A. A rare case of large mediastinal germ cell tumor detected by echocardiography. Int J Cardiol 2005; 101: 159-161.

2. Farheen B, Shagufta Y, Nishat A, Nazoora K, Shah FA. Benign mediastinal teratoma with intrapulmonary and bronchial ropture presenting with recurrent hemoptysis. Iran J Radiol 2013; 10: $86-89$.

3. Stella F, Davoli F. Giant mediastinal mature teratoma with increased exocrine pancreatic activity presenting in a young woman: a case report. J Med Case Reports 2011; 5: 238.

4. Kallis P, Treasure T, Holmes SJ, Grffiths M. Exocrine pancreatic function in mediastinal teratoma: an aid to preoperative diagnosis? Ann Thorac Surg 1992; 54: 741-743.

5. Bordi C, De Vita O, Pollice L. Full pancreatic endocrine differentiation in a mediastinal teratoma. Hum Pathol 1985; 16 : 941-944. 